\title{
Management of severe frostbite with iloprost, alteplase and heparin: a Yukon case series
}

\author{
Alexander Poole MD, Josianne Gauthier MScPharm, Mira MacLennan MD
}

Abstract

Background: We identified the need to modernize frostbite management in our northern centre and implemented a treatment protocol in 2015. Our aim was to describe the clinical course of patients presenting to the hospital since the implementation of the protocol.

Methods: This was a retrospective case series from Whitehorse General Hospital, Whitehorse, Yukon Territory, Canada. We reviewed the charts of patients who presented to the hospital with grade 2-4 frostbite and were treated as per our protocol between Feb. 9, 2015, and Feb. 8, 2020. Patients with grade 2-4 frostbite received iloprost; in addition, those with grade 4 frostbite received alteplase and heparin. We determined the number of digits affected and salvaged, and the time from presentation to the emergency department to treatment initiation. We also examined patients' demographic characteristics, predisposing events, frostbite severity and adverse drug reactions.

Results: In 22 patients treated for grade 2-4 frostbite, 142 digits were affected: 59 with grade 2 frostbite, 25 with grade 3 frostbite and 58 with grade 4 frostbite; of the 142, 113 (79.6\%) were salvaged. All 29 digits amputated had grade 4 frostbite. The mean time from presentation to iloprost initiation was reduced from 32.9 hours in 2015 to 3.0 hours in 2020 . Sports (10 cases [45\%]) and alcohol use $(6[27 \%])$ were the most common precipitating events, with alcohol use tending to result in more severe injury (grade 4 in 5 of 6 cases). Adverse reactions with iloprost (e.g., headache) were common but mild. Adverse reactions with alteplase (e.g., bleeding) were less common but of greater clinical significance.

Interpretation: Over the study period, our protocol contributed to improvement in frostbite care at our institution, resulting in a digit salvage rate comparable to other published results. Our 5-year experience shows that advanced medical care of frostbite can be achieved, even at a rural centre.

F rostbite can occur in cold climates, affecting a range of patients from those who are homeless or unwell, to athletes in extreme sports events. The impact of severe frostbite can be debilitating. Frostbite injuries are a result of an initial extracellular freezing injury followed by a reperfusion injury due to vasoconstriction and microthrombosis in affected tissues. ${ }^{1}$ The severity of injuries can range from mild to severe. A grading system developed by Cauchy and colleagues ${ }^{2,3}$ describes grade 1 to grade 4 frostbite. The higher the grade, the more proximal the cyanotic changes in the digits, and the higher the amputation rate.

The foundations of frostbite treatment have included rapid rewarming ${ }^{4}$ and antithromboxane agents such as aloe vera for topical use and ibuprofen. ${ }^{5}$ More recently, vasodilators and thrombolytics have been promoted to address the reperfusion injury caused by vasoconstriction and thrombosis. ${ }^{6-28}$ Beginning in the 1990s, a growing body of evidence has popularized the use of iloprost in frostbite, first in Europe and now in Canada. $6,11,15,16,21,24,27$ Iloprost, a synthetic prostacyclin analogue, is a potent vasodilator that inhibits platelet aggregation and enhances fibrinolytic activity by releasing endogenous tissue plasminogen activator. ${ }^{29}$ To our knowledge, there is a single randomized controlled trial published to date showing the benefit of iloprost combined to alteplase and heparin. ${ }^{11}$

We developed and implemented a frostbite treatment protocol and preprinted orders at our institution based on the best available evidence in February 2015. The aim of the present study was to describe the demographic characteristics, treatment course and clinical outcomes of patients treated as per our protocol.

Competing interests: Josianne Gauthier is employed by the Whitehorse General Hospital (Yukon Hospital Corporation). No other competing interests were declared.

This article has been peer reviewed.

Correspondence to: Alexander Poole, alexjpoole@mac.com

CMAJ Open 2021. DOI:10.9778/cmajo.20200214 


\section{Methods}

\section{Study setting, design and participants}

This was a single-centre retrospective series of cases in Whitehorse, Yukon Territory, Canada. The Yukon extends from $60^{\circ} \mathrm{N}$ to almost $70^{\circ} \mathrm{N}$ latitude. It has expected temperatures below $-40^{\circ} \mathrm{C}$ every winter from November to April. Whitehorse, the capital, attracts elite athletes for extreme outdoor winter sporting events. Given this environment, we identified the need to modernize our frostbite management and implemented a treatment protocol and preprinted orders based on the best available evidence in February 2015. We published our first 2 cases in $2016 .^{15}$

We reviewed the charts of all consecutive patients aged 18 years or more who presented to Whitehorse General Hospital, a rural and remote hospital, with grade 2-4 frostbite and were treated with iloprost as per our protocol between Feb. 9, 2015, and Feb. 8, 2020. Patients who presented 72 hours or more after rewarming were excluded.

\section{Yukon Frostbite Protocol}

After implementation of the frostbite protocol (Figure 1), several education sessions were offered to the hospital staff and Yukon community health care providers. Patients diagnosed with frostbite in the emergency department underwent rapid rewarming of the affected extremities if not previously rewarmed. Frostbite grade was established with the visual grading system developed by Cauchy and colleagues. ${ }^{2,3}$ Cases of grade 1-4 frostbite were managed with daily hydrotherapy, débridement and aspiration of clear blisters, topical administration of aloe vera (Aloe Vesta ointment, ConvaTec), scheduled oral ibuprofen treatment and pantoprazole.

Patients with grade 2-4 frostbite who presented within 72 hours of rewarming received iloprost (Ilomedin [Bayer]), obtained through Health Canada's Special Access Programme, diluted at a standard concentration of $0.2 \mu \mathrm{g} / \mathrm{mL}$ and initiated at a rate of $10 \mathrm{~mL} / \mathrm{h}$ intravenous infusion to a maximum rate according to the patient's weight for a total of $6 \mathrm{~h})$. Patients with grade 4 frostbite who presented within 24 hours of rewarming also received alteplase $(15 \mathrm{mg}$ given intravenously over $2 \mathrm{~min}$, then $0.75 \mathrm{mg} / \mathrm{kg}$ [maximum $50 \mathrm{mg}$ ] over $30 \mathrm{~min}$, then $0.5 \mathrm{mg} / \mathrm{kg}$ [maximum $35 \mathrm{mg}$ ] over $60 \mathrm{~min}$ ) and heparin (60 units/ $\mathrm{kg}$ given intravenously, then 12 units $/ \mathrm{kg}$ per hour for 6 hours, then adjusted according to the partial thromboplastin time for $72 \mathrm{~h}$ ) unless contraindicated.

After the protocol was implemented, we made 4 substantial changes: 1) treat grade 2 cases with iloprost, 2) treat patients with grade 2 or 3 frostbite in an ambulatory setting, 3) decrease the alteplase dosage (to $0.15 \mathrm{mg} / \mathrm{kg}$ over $15 \mathrm{~min}$, then intravenous infusion of $0.15 \mathrm{mg} / \mathrm{kg}$ per hour for $6 \mathrm{~h}$ ) and 4) include pantoprazole.

\section{Data collection}

The computer pharmacy system, Meditech, was used to identify patients who had received iloprost for frostbite. The patients' medical records were reviewed independently by

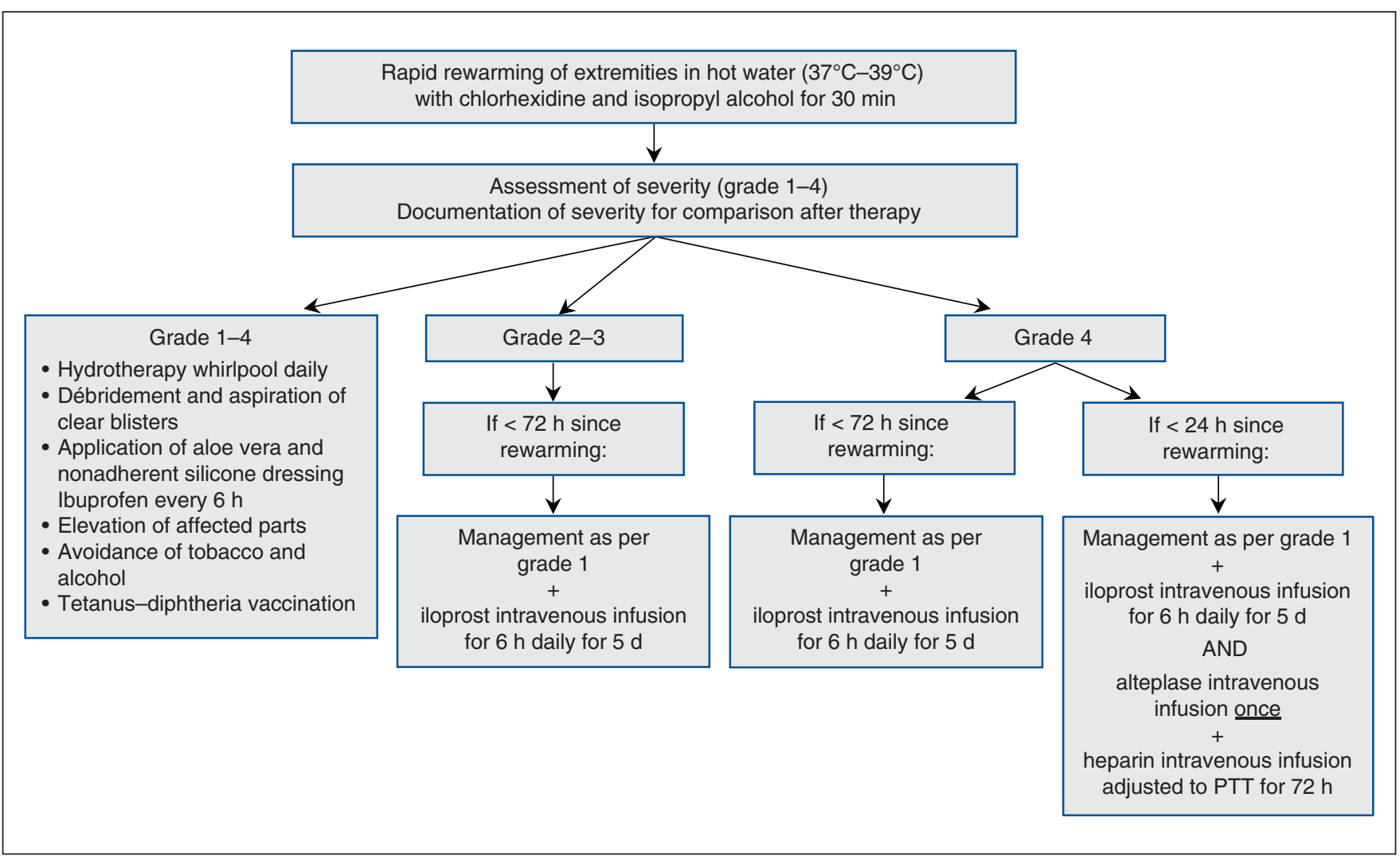

Figure 1: Yukon Frostbite Protocol. Note: PTT = partial thromboplastin time. 
2 reviewers (A.P. and J.G.). We developed a data abstraction tool in Microsoft Excel; data extracted included patients' demographic characteristics, frostbite severity, digits affected, and factors known or suspected to affect frostbite treatment outcomes: ${ }^{1,22,30}$ patients' comorbidities, local environmental temperature (based on Environment Canada data [https:// climate.weather.gc.ca/]), body temperature, duration of cold exposure, predisposing event, time from presentation to the emergency department to rewarming, and time from presentation to treatment. Adverse drug reactions were also noted. Discrepancies in data collection were evaluated by a third independent reviewer (M.M.). When discrepancies still existed, the 3 reviewers met to review chart data further and achieve consensus.

\section{Outcomes}

The primary outcome was the number of digits salvaged compared to the number of digits affected. We also examined whether there was a correlation between the environmental temperature or duration of cold exposure with frostbite severity, adverse drug reactions and time from presentation to treatment initiation.

\section{Statistical analysis}

We performed descriptive statistical analysis using Excel, version 16.16.22. We performed 1-way analysis of variance using SPSS software, build 1.0.0.1347 (IBM Corporation).

\section{Ethics approval}

The Yukon Frostbite Protocol was approved by the Yukon Hospital Corporation Ethics Committee before implementation. The preprinted orders were approved by the Pharmacy and Therapeutics Committee and Medical Advisory Committee. Permission to review the patients' medical records was obtained from the Yukon Hospital Corporation Ethics Committee.

\section{Results}

From Feb. 9, 2015, to Feb. 8, 2020, Whitehorse General Hospital received and treated 23 patients with a diagnosis of grade 2-4 frostbite. One patient was excluded from this case series as there was uncertainty as to whether an acute frostbite injury had occurred. This patient presented with fissured and blackened toes that were ischemic and dusky. The patient was transferred to a referral hospital out of territory owing to other medical conditions requiring a higher level of care. The demographic and clinical characteristics of the remaining 22 patients are summarized in Table 1.

Of the 22 cases, $19(86 \%)$ occurred at a temperature of $-21^{\circ} \mathrm{C}$ or colder; most cases occurred between $-21^{\circ} \mathrm{C}$ and $-30^{\circ} \mathrm{C}$. There was no significant correlation between temperature and frostbite severity $(p=0.4)$ (Figure 2$)$. The exact duration of cold exposure was difficult to determine from patients' histories but ranged from as short as 45 minutes to 64 hours. There was no significant correlation between the duration of cold exposure and frostbite severity $(p=0.4)$. Pre-

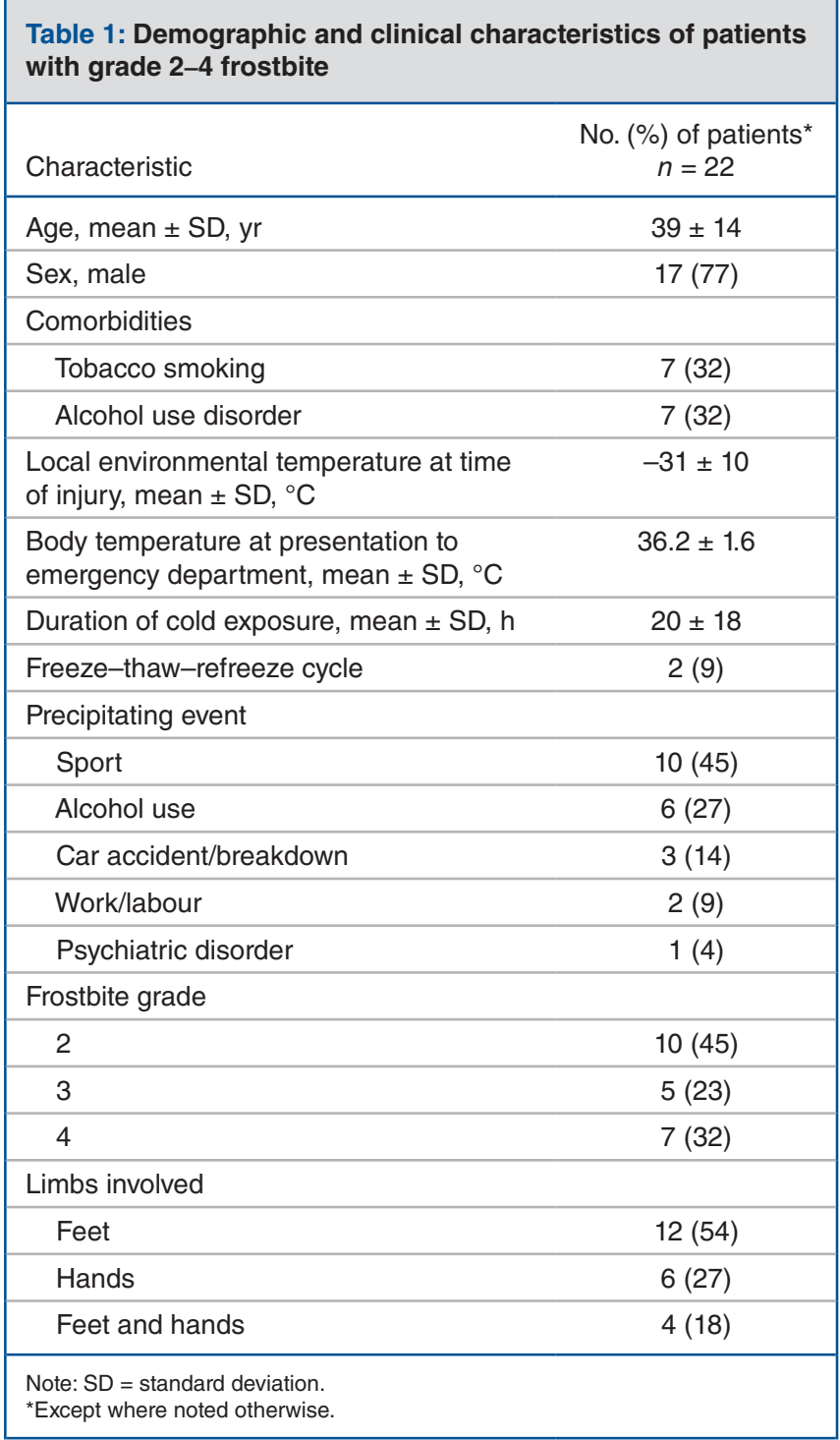

disposing events leading to frostbite injury fell into 3 main categories: winter sports, alcohol use, and car accident or breakdown (Table 1). Winter sports precipitated 10 cases (45\%); 8 of the 10 patients were participating in the Yukon Arctic Ultra, an endurance running race that takes place every year in February. Alcohol use precipitated 6 cases (27\%), which showed a trend toward more severe injury, with 5 of the 6 cases being grade 4 (Figure 3).

\section{Treatment and adverse drug reactions}

The patients were managed and followed by the surgical team. Follow-up included in-person clinic visits, telehealth consultations or emails, and photographs. The treatment details and adverse drug reactions are summarized in Table 2. Although the majority of patients (13 [59\%]) were rewarmed rapidly in the emergency department, 9 patients $(41 \%)$ had passive rewarming before presentation to Whitehorse General Hospital. None of the passively rewarmed patients required amputation. 


\section{Research}

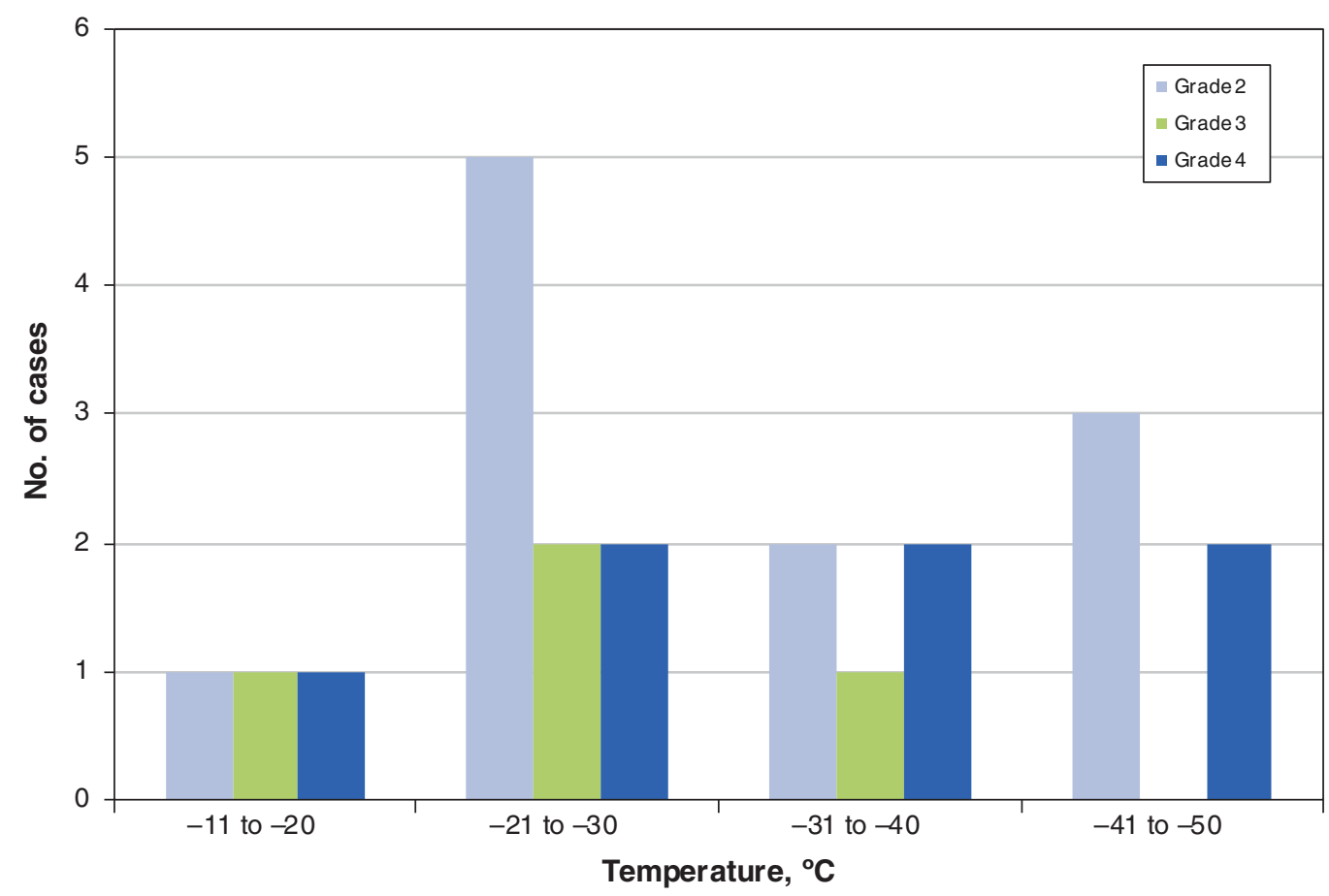

Figure 2: Frostbite severity by local environmental temperature at the time of the injury.

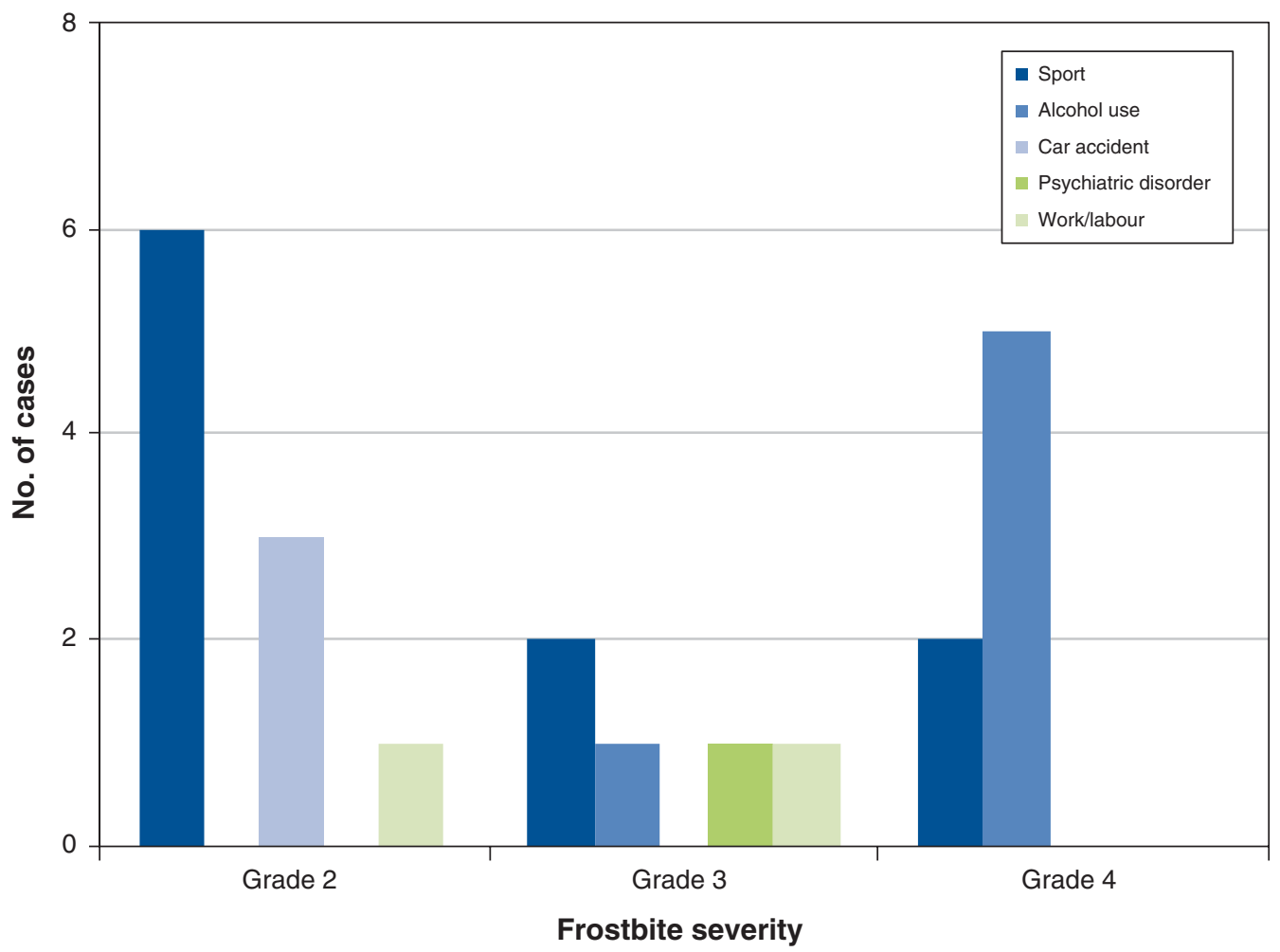

Figure 3: Frostbite severity by precipitating event. 


\begin{tabular}{|c|c|}
\hline \multicolumn{2}{|l|}{ Table 2: Treatment and adverse drug reactions } \\
\hline Variable & $\begin{array}{l}\text { No. }(\%) \text { of } \\
\text { patients }\end{array}$ \\
\hline \multicolumn{2}{|l|}{ Treatment } \\
\hline Rapid rewarming & $13(59)$ \\
\hline Iloprost only & $9(41)$ \\
\hline Iloprost, alteplase and heparin & $4(18)$ \\
\hline Passive rewarming & $9(41)$ \\
\hline Iloprost only & $8(36)$ \\
\hline Iloprost, alteplase and heparin & $1(4)$ \\
\hline \multicolumn{2}{|l|}{ Treatment adjunct } \\
\hline Topically administered aloe vera & $19(86)$ \\
\hline Scheduled orally administered ibuprofen & $22(100)$ \\
\hline \multicolumn{2}{|l|}{ No. of iloprost doses received } \\
\hline 1 & $2(9)$ \\
\hline 3 & $3(14)$ \\
\hline 4 & $1(5)$ \\
\hline 5 & $16(73)$ \\
\hline \multicolumn{2}{|l|}{ Alteplase dosing $(n=5)$} \\
\hline $\begin{array}{l}15 \mathrm{mg} \text { intravenously over } 2 \mathrm{~min} \text {, then } \\
0.75 \mathrm{mg} / \mathrm{kg} \text { (maximum } 50 \mathrm{mg} \text { ) over } 30 \mathrm{~min} \text {, } \\
\text { then } 0.5 \mathrm{mg} / \mathrm{kg} \text { (maximum } 35 \mathrm{mg} \text { ) over } \\
60 \mathrm{~min}\end{array}$ & $1(20)$ \\
\hline $\begin{array}{l}0.15 \mathrm{mg} / \mathrm{kg} \text { over } 15 \mathrm{~min} \text {, then } 0.15 \mathrm{mg} / \mathrm{kg} \text { per } \\
\text { hour intravenously for } 6 \mathrm{~h}^{*}\end{array}$ & $4(80)$ \\
\hline \multicolumn{2}{|l|}{ Adverse drug reactions } \\
\hline \multicolumn{2}{|l|}{ Iloprost } \\
\hline Headache & $11(50)$ \\
\hline Flushing & $8(36)$ \\
\hline Tachycardia (heart rate $>100$ beats/min) & $8(36)$ \\
\hline Nausea & $6(27)$ \\
\hline Vomiting & $2(9)$ \\
\hline Dizziness & $2(9)$ \\
\hline Bleeding & $1(4)$ \\
\hline Hypotension (blood pressure < 90/50 mm Hg) & $1(4)$ \\
\hline \multicolumn{2}{|l|}{ Alteplase $(n=5)$} \\
\hline Acute intramuscular hemorrhage & $1(20)$ \\
\hline Bleeding from wounds & $1(20)$ \\
\hline
\end{tabular}

Sixteen patients $(73 \%)$ had adverse reactions with iloprost, most commonly headache (11 [50\%]), which was managed with a decrease in the infusion rate. Flushing (8 patients [36\%]), tachycardia (8 [36\%]) and nausea (6 [27\%]) were the next most common reactions and were managed symptomatically. One patient (4\%) had frank bleeding below the eye after iloprost infusion, but this may have been due to a recent physical altercation. This patient was also receiving venous thromboembolism prophylaxis with dalteparin. Another patient receiving iloprost daily developed a perforated duodenal ulcer on hospital day 5. The patient was receiving scheduled ibuprofen and was not receiving any gastric-protecting agent.

Five patients received alteplase and heparin in addition to iloprost, 2 of whom had adverse reactions: an acute intramuscular hemorrhage in 1 patient and bleeding from wounds in the other; both required blood transfusion.

\section{Outcomes}

There were 142 affected digits, 59 with grade 2 frostbite, 25 with grade 3 frostbite and 58 with grade 4 frostbite. All digits with grade 2 or 3 frostbite were salvaged, as were 29 $(50 \%)$ of those with grade 4 frostbite, for an overall salvage rate of $79.6 \%(113 / 142)$. Four patients required amputation. All 29 digits amputated had grade 4 frostbite; the majority of digits amputated (19) were from 1 patient.

\section{Time to treatment}

The mean time from emergency department presentation (triage) to rewarming was 2.4 (standard deviation [SD] 3.8) hours. The mean time from emergency department presentation to iloprost initiation was reduced from 32.9 (SD 21.9) hours in 2015 to 3.0 (SD 2.1) hours in 2020 (Figure 4). There were only 2 years with grade 4 cases that met criteria for alteplase treatment, 2017 and 2018. For these 2 years, the mean time from emergency department triage to alteplase initiation decreased from 7.4 (SD 3.7) hours in 2017 to 4.6 (SD 0.1 ) hours in 2018.

\section{Interpretation}

Among patients treated with a protocol including iloprost for grade 2-4 frostbite, and alteplase and heparin for grade 4 frostbite, our overall digit salvage rate was $80 \%$ (amputation rate $20 \%$ ). Grade 2 and 3 cases had a salvage rate of $100 \%$, and grade 4 cases had a salvage rate of $50 \%$. The risk of amputation without iloprost or thrombolysis has been reported to be $31 \%$ for grade 2 frostbite, $67 \%$ for grade 3 frostbite and $98 \%-100 \%$ for grade 4 frostbite. ${ }^{2}$ Our digit salvage rate is consistent with that reported by Lindford and colleagues, ${ }^{21}$ who obtained a rate of $74.8 \%$ using iloprost or alteplase in their series of 20 cases.

Iloprost was well tolerated in our series, with the most common adverse reaction being headache, in $50 \%$ of patients. Only 1 patient receiving iloprost had bleeding, from an altercation injury, but this patient was also receiving an anticoagulant for venous thromboembolism prophylaxis, which may have contributed to the bleeding. Another patient receiving iloprost developed a perforated duodenal ulcer; this was thought to be related to the use of ibuprofen $(2400 \mathrm{mg} / \mathrm{d}$ for $5 \mathrm{~d})$ without a gastric-protecting agent. Of the 5 patients who received alteplase and heparin, 2 had bleeding requiring blood transfusion. This was an expected risk associated with thrombolytic and anticoagulant use. ${ }^{23}$

In our series, more cases were related to outdoor sports than to psychiatric illness or alcohol use. This is likely different from what would be expected in an urban centre. We had 


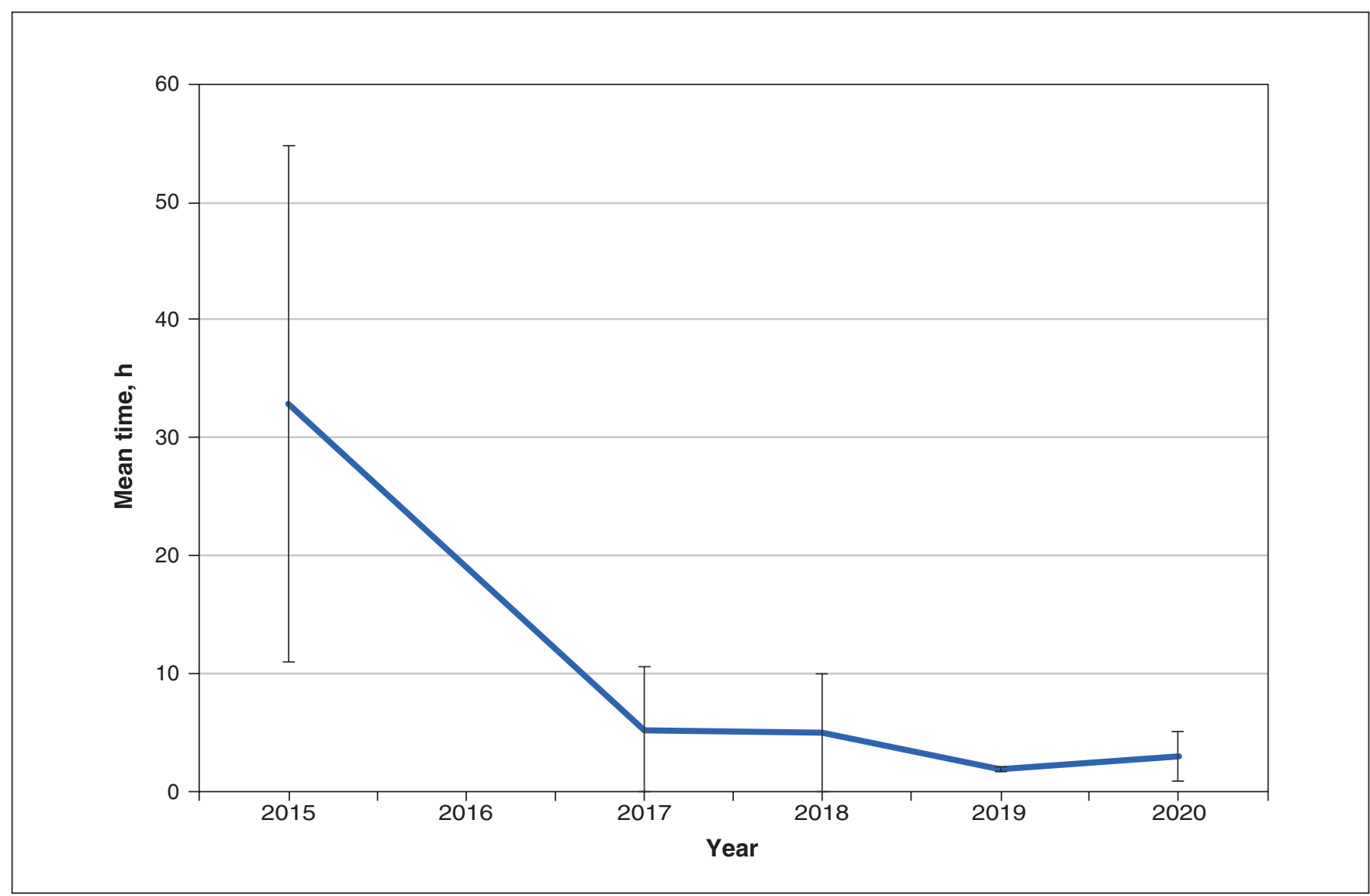

Figure 4: Mean time from emergency department presentation to iloprost treatment. No iloprost was given in 2016. Error bars = standard deviation.

no cases of frostbite at temperatures above $-14^{\circ} \mathrm{C}$, with $86 \%$ of cases occurring at temperatures below $-20^{\circ} \mathrm{C}$. This is consistent with the findings of Juopperi and colleagues, ${ }^{30}$ who showed that, in Finland, the annual incidence of frostbite started to increase at temperatures below $-15^{\circ} \mathrm{C}$ and was considerable at temperatures below $-20^{\circ} \mathrm{C}$. The majority of our cases occurred below the temperature at which Boles and colleagues ${ }^{31}$ found an increased risk of tissue loss, $-23^{\circ} \mathrm{C}$.

We observed a decrease in time to initiation of treatment over our study period. As other authors have observed, ${ }^{26}$ we found that having a formal protocol and preprinted orders led to a decrease in treatment delay. Great efforts were made to educate front-line providers in the emergency department, community hospitals and health care centres. As familiarity with frostbite recognition, grading and management improved, time to treatment initiation decreased. This is important progress, as Nygaard and colleagues ${ }^{22}$ found that the time between rewarming and thrombolytic therapy (warm ischemia time) was a critical window, with each hour of delay resulting in a decrease in digit salvage of $28 \%$.

We made 4 substantial changes to our protocol after it was implemented. To avoid undertreatment or delayed treatment of grade 3 frostbite, and given how well iloprost was tolerated, we decided to treat grade 2 frostbite with iloprost. This arose from the difficulty distinguishing some grade 3 cases from grade 2 cases early on. We also treated many patients with grade 2 or 3 frostbite in an ambulatory setting (outpatient therapy in the emergency department or critical care department) for convenience. The third change was a decrease in the alteplase dosage. We initially used the dosing of Cauchy and colleagues ${ }^{11}$ but revised our dosing in February 2017 based on the experience of the Hennepin County Medical Center in Minneapolis. ${ }^{22}$ The revised alteplase dosing yields a smaller total dose (16\% less in an $80-\mathrm{kg}$ patient). We believed that administration would be simpler and there would be a lower risk of adverse drug events. We also revised our protocol in April 2018 to include the proton-pump inhibitor pantoprazole after 1 patient experienced a perforated duodenal ulcer.

Given the risks associated with use of alteplase, this drug should be reserved for patients at highest risk for amputation. ${ }^{23}$ Iloprost can be used in patients with any level of cyanosis and risk of digit loss (grade $2-4$ frostbite), and can be effective up to 72 hours after rewarming. ${ }^{24}$ Iloprost is not currently available commercially in Canada and can be obtained only through Health Canada's Special Access Programme. We identified a single published case report on the use of the prostacyclin epoprostenol in a patient with frostbite, at a US institution without access to iloprost. ${ }^{26}$ Because of the limited evidence on the use of epoprostenol in frostbite management, we suggest that iloprost be used instead. We encourage Canadian hospitals in regions where people are at risk for cold injury to obtain access to iloprost for use in frostbite. 


\section{Limitations}

Our study is limited by its retrospective nature and the limitations of chart data extraction. Rewarming outside of the hospital setting was uncontrolled, and the rewarming method for those patients could not be evaluated. Our protocol was changed over the course of the 5-year study period. Because there was no control group, our case series did not allow us to verify the benefit of iloprost, alteplase and heparin compared to no pharmacologic intervention. Our case series also did not allow us to determine whether the combination of iloprost and alteplase provides additional benefit compared to either agent alone. Ideally, this question would be addressed in a randomized controlled trial; a national registry could serve to collect data on all frostbite cases, standardize data selection and show whether there are enough cases to power such a study sufficiently. Finally, as grading of frostbite relies on subjective clinical interpretation, a study validating clinical grading compared to advanced imaging would be beneficial.

\section{Conclusion}

Our 5-year experience shows that advanced medical care of severe frostbite can be achieved, even at a rural centre. Our protocol reduced time to treatment and resulted in a digit salvage rate comparable to other published results. Adverse reactions with iloprost were common but mild. Adverse reactions with alteplase were less common but of greater clinical import. We believe that a frostbite protocol with a visual grading system, preprinted orders and education of health care providers contributed to improved frostbite care at our institution.

\section{References}

1. Mohr WJ, Jenabzadeh K, Ahrenholz DH. Cold injury. Hand Clin 2009;25: 481-96.

2. Cauchy E, Chetaille E, Marchand V, et al. Retrospective study of 70 cases of severe frostbite lesions: a proposed new classification scheme. Wilderness Environ Med 2001;12:248-55.

3. Cauchy E, Davis CB, Pasquier M, et al. A new proposal for management of severe frostbite in the austere environment. Wilderness Environ Med 2016;27: 92-9.

4. Mills WJ Jr, Whaley R, Fish W. Frostbite: experience with rapid rewarming and ultrasonic therapy. Alaska Med 1960;2:1-4.

5. Heggers JP, Robson MC, Manavalen K, et al. Experimental and clinical observations on frostbite. Ann Emerg Med 1987;16:1056-62.

6. Groechenig E. Treatment of frostbite with iloprost. Lancet 1994;344:1152-3.

7. Twomey JA, Peltier GL, Zera RT. An open-label study to evaluate the safety and efficacy of tissue plasminogen activator in treatment of severe frostbite. 7 Trauma 2005;59:1350-4, discussion 1354-5.

8. Bruen KJ, Ballard JR, Morris SE, et al. Reduction of the incidence of amputation in frostbite injury with thrombolytic therapy. Arch Surg 2007;142:546-51, discussion 551-3.

9. Sheridan RL, Goldstein MA, Stoddard FJ Jr, et al. Case records of the Massachusetts General Hospital. Case 41-2009. A 16-year-old boy with hypothermia and frostbite. N Engl F Med 2009;361:2654-62.

10. Saemi AM, Johnson JM, Morris CS. Treatment of bilateral hand frostbite using transcatheter arterial thrombolysis after papaverine infusion. Cardiovasc Intervent Radiol 2009;32:1280-3.

11. Cauchy E, Cheguillaume B, Chetaille E. A controlled trial of a prostacyclin and rt-PA in the treatment of severe frostbite. N Engl F Med 2011;364:189-90.

12. Johnson AR, Jensen HL, Peltier G, et al. Efficacy of intravenous tissue plasminogen activator in frostbite patients and presentation of a treatment protocol for frostbite patients. Foot Ankle Spec 2011;4:344-8.

13. Gross EA, Moore JC. Using thrombolytics in frostbite injury. 7 Emerg Trauma Shock 2012;5:267-71.

14. Ibrahim AE, Goverman J, Sarhane KA, et al. The emerging role of tissue plasminogen activator in the management of severe frostbite. 7 Burn Care Res 2015; 36:e62-6.
15. Poole A, Gauthier J. Treatment of severe frostbite with iloprost in northern Canada. CMA7 2016;188:1255-8.

16. Haik J, Brown S, Liran A, et al. Deep frostbite: the question of adjuvant treatment. Isr Med Assoc 7 2016;18:56-7.

17. Tavri S, Ganguli S, Bryan RG Jr, et al. Catheter-directed intraarterial thrombolysis as part of a multidisciplinary management protocol of frostbite injury. $\mathcal{F}$ Vasc Interv Radiol 2016;27:1228-35.

18. Gonzaga T, Jenabzadeh K, Anderson CP, et al. Use of intra-arterial thrombolytic therapy for acute treatment of frostbite in 62 patients with review of thrombolytic therapy in frostbite. 7 Burn Care Res 2016;37:e323-34.

19. Wexler A, Zavala S. The use of thrombolytic therapy in the treatment of frostbite injury. 7 Burn Care Res 2017;38:e877-81.

20. Patel N, Srinivasa DR, Srinivasa RN, et al. Intra-arterial thrombolysis for extremity frostbite decreases digital amputation rates and hospital length of stay. Cardiovasc Intervent Radiol 2017;40:1824-31.

21. Lindford A, Valtonen J, Hult M, et al. The evolution of the Helsinki frostbite management protocol. Burns 2017;43:1455-63.

22. Nygaard RM, Lacey AM, Lemere A, et al. Time matters in severe frostbite: assessment of limb/digit salvage on the individual patient level. 7 Burn Care Res 2017;38:53-9.

23. Jones LM, Coffey RA, Natwa MP, et al. The use of intravenous tPA for the treatment of severe frostbite. Burns 2017;43:1088-96.

24. Pandey P, Vadlamudi R, Pradhan R, et al. Case report: severe frostbite in extreme altitude climbers: the Kathmandu iloprost experience. Wilderness Environ Med 2018;29:366-74.

25. Al Yafi MN, Danino MA, Izadpanah A, et al. Using intra-arterial tPA for severe frostbite cases. An observational comparative retrospective study. 7 Burn Care Res 2019;40:907-12.

26. Khan SL, Parikh R, Mooncai T, et al. Barriers to frostbite treatment at an academic medical center. Am 7 Emerg Med 2019;37:1601.e3-5.

27. Jud P, Hafner F, Brodmann M. Frostbite of the hands after paragliding: a chilling experience. Lancet 2019;394:2282.

28. Heard J, Shamrock A, Galet C, et al. Thrombolytic use in management of frostbite injuries: eight year retrospective review at a single institution. $7 \mathrm{Burm}$ Care Res 2020;41:722-6.

29. Musiał J, Wilczyńska M, Sładek K, et al. Fibrinolytic activity of prostacyclin and iloprost in patients with peripheral arterial disease. Prostaglandins 1986;31: 61-70.

30. Juopperi K, Hassi J, Ervasti O, et al. Incidence of frostbite and ambient temperature in Finland, 1986-1995. A national study based on hospital admissions. Int 7 Circumpolar Health 2002;61:352-62.

31. Boles R, Gawaziuk JP, Cristall N, et al. Pediatric frostbite: a 10-year singlecenter retrospective study. Burns 2018;44:1844-50.

Affiliations: Whitehorse General Hospital (Poole), Whitehorse, Yukon; Department of Surgery (Poole), Cumming School of Medicine, University of Calgary, Calgary, Alta.; Whitehorse General Hospital (Gauthier), Yukon Hospital Corporation, Whitehorse, Yukon; University of Saskatchewan (Gauthier), Saskatoon, Sask.; Department of Surgery (MacLennan), McMaster University, Hamilton, Ont.; Harvard T.H. Chan School of Public Health (MacLennan), Boston, Mass.

Contributors: Alexander Poole and Josianne Gauthier conceived of and designed the study, and obtained the data. All of the authors analyzed and interpreted the data, drafted the manuscript and revised it critically for important intellectual content, approved the final version to be published and agreed to be accountable for all aspects of the work.

Funding: The authors did not receive funding for this article. They also did not receive funding or any form of compensation to use the drugs cited in this article. The drugs were provided by the hospital pharmacy department for inpatient use. Iloprost was obtained at no cost from Bayer through Health Canada's Special Access Programme.

Content licence: This is an Open Access article distributed in accordance with the terms of the Creative Commons Attribution (CC BY-NCND 4.0) licence, which permits use, distribution and reproduction in any medium, provided that the original publication is properly cited, the use is noncommercial (i.e., research or educational use), and no modifications or adaptations are made. See: https://creativecommons.org/licenses/ by-nc-nd/4.0/.

Data sharing: The data from this case series, the Yukon Frostbite Protocol and preprinted orders are available from the corresponding author on request.

Supplemental information: For reviewer comments and the original submission of this manuscript, please see www.cmajopen.ca/content/9/2/ E585/suppl/DC1. 